\title{
Regional Variation on Rates of Bronchopulmonary Dysplasia and Associated Risk Factors
}

\author{
María Ximena Rojas, ${ }^{1}$ Mario Augusto Rojas, ${ }^{2}$ Juan Manuel Lozano, ${ }^{3}$ \\ Martín Alonso Rondón, ${ }^{1}$ and Laura Patricia Charry ${ }^{1}$ \\ ${ }^{1}$ Department of Clinical Epidemiology and Biostatistics, School of Medicine, Pontificia Universidad Javeriana, \\ Bogotá 110001, D.C., Colombia \\ ${ }^{2}$ Division of Neonatology, Wake Forest University School of Medicine, Winston-Salem, NC, USA \\ ${ }^{3}$ Division of Research and Information, College of Medicine, Florida International University, Miami, FL, USA \\ Correspondence should be addressed to María Ximena Rojas, mxrojas@javeriana.edu.co
}

Received 6 March 2012; Accepted 9 May 2012

Academic Editors: G. J. Casimir, D. L. Jeppesen, S. K. Patole, and T. F. Yeh

Copyright ( $) 2012$ María Ximena Rojas et al. This is an open access article distributed under the Creative Commons Attribution License, which permits unrestricted use, distribution, and reproduction in any medium, provided the original work is properly cited.

\begin{abstract}
Background. An abnormally high incidence (44\%) of bronchopulmonary dysplasia with variations in rates among cities was observed in Colombia among premature infants. Objective. To identify risk factors that could explain the observed high incidence and regional variations of bronchopulmonary dysplasia. Study Design. A case-control study was designed for testing the hypothesis that differences in the disease rates were not explained by differences in city-of-birth specific population characteristics or by differences in respiratory management practices in the first 7 days of life, among cities. Results. Multivariate analysis showed that premature rupture of membranes, exposure to mechanical ventilation after received nasal CPAP, no surfactant exposure, use of rescue surfactant (instead of early surfactant), PDA, sepsis and the median daily $\mathrm{FIO}_{2}$, were associated with a higher risk of dysplasia. Significant differences between cases and controls were found among cities. Models exploring for associations between city of birth and dysplasia showed that being born in the highest altitude city (Bogotá) was associated with a higher risk of dysplasia (OR 1.82 95\% CI 1.31-2.53). Conclusions. Bronchopulmonary dysplasia was manly explained by traditional risk factors. Findings suggest that altitude may play an important role in the development of this disease. Prenatal steroids did not appear to be protective at high altitude.
\end{abstract}

\section{Introduction}

Despite all the advances in the care of premature infants with respiratory distress syndrome (RDS), including the use of antenatal steroids and early management with surfactant, bronchopulmonary dysplasia (BPD) continues to be a major cause of chronic morbidity among this population. There are large variations in the incidence and severity of this disease. According to the National Institutes of Health of USA (NICHD) consensus [1], mild BPD is defined as a need for supplemental oxygen for $\geq 28$ days at 36 weeks postmenstrual age (wPMA) or discharge, moderate BPD as supplemental oxygen for $\geq 28$ days plus treatment with $<30 \%$ oxygen at 36 wPMA, and severe BPD as supplemental oxygen for $\geq 28$ days plus $\geq 30 \%$ oxygen and/or positive pressure at 36 wPMA. Currently, the estimated incidence of BPD defined as need for supplemental oxygen at 36 wPMA in the United States is approximately $30 \%$ for premature infants with a birth weight $<1000$ grams and $<7 \%$ in infants with a birth weight $>1250$ grams or who were at least 30 weeks of gestation at birth $[1,2]$.

Previous epidemiological studies have identified prematurity, oxygen toxicity, and mechanical ventilation as major risk factors associated with BPD [3-7]. Additional risk factors include perinatal or postnatal infection/inflammation [8-12], pulmonary edema resulting from patent ductus arteriosus (PDA) $[12,13]$ or excess fluid administration [7, $14,15]$, vitamin A deficiency [16], early adrenal insufficiency [17], and, more recently, genetic polymorphism [18].

There is little information about trends in the epidemiology and pathogenesis of BPD in developing countries. 
The most recent report of the incidence of BPD in Latin America comes from the NEOCOSUR Neonatal Group study in 1,825 very-low-birth-weight (VLBW) infants born in sixteen hospitals from Argentina, Chile, Paraguay, Peru, and Uruguay [19]. The authors found an incidence of BPD (oxygen requirement at 28 days of life with chronic radiographic changes) of $24.4 \%$. A randomized controlled trial of early bubble nasal continuous positive airway pressure (nCPAP) and surfactant in premature infants conducted in three different cities in Colombia [20] found an incidence of BPD (defined as supplemental oxygen requirement at 36 wPMA) twice as large (44\%) as the one observed in less mature premature infants in developed countries or in the NEOCOSUR study. The Colombian trial also revealed significant variations in BPD rates among participating cities: Bogotá 50\%, Cali 18\%, and Bucaramanga 13\%. These cities have different characteristics, the most important being altitude: Bogotá is 2600 meters above sea level (masl), Bucaramanga 959 masl, and Cali 956 masl.

We conducted this study with the aim of identifying environmental, maternal, infant, and therapeutic risk factors associated with BPD in Colombia. We also tested the hypothesis that differences in BPD rates were not explained by differences in city-of-birth-specific population characteristics or by differences in respiratory management practices during the first seven days of infants' life among cities.

\section{Materials/Subjects and Methods}

This is a nested case-control study based on data collected as part of a multicenter randomized controlled trial carried out by the Colombian Neonatal Research Network in eight neonatal intensive care units (NICUs) located in three cities (Bogotá, Bucaramanga, and Cali) in Colombia. A detailed description of this trial has been published [20]. Briefly, premature infants born between 27 and 31 weeks of gestation with clinical evidence of respiratory distress during the first hour of life, and who did not require intubation as part of their initial resuscitation and stabilization, were placed on bubble nCPAP and then randomized to receive very early surfactant therapy through transient intubation followed by nCPAP or to expectant management on nCPAP alone. A total of 279 premature infants were enrolled in the trial from January 1, 2004 to December 31, 2006. All study sites provided comprehensive continuous care for critically ill neonates and were staffed by trained nurses and specialized physicians with fully equipped and modern neonatal units. Prenatal and neonatal data were collected prospectively until death or discharge. Additional data were collected at 36 wPMA.

The neonatal survival for the population included in the RCT was $90.7 \%$. Neonatal mortality rates were similar among cities. For the present study, all analyses were limited to infants who survived to 36 wPMA. Bronchopulmonary dysplasia was defined as the need for supplemental oxygen for $\geq 28$ days at 36 wPMA [1]. The target oxygen arterial saturation $\left(\mathrm{SaO}_{2}\right)$ was $92 \%$ for infants treated in Bogota and $96 \%$ for those treated in other cities. Given the high altitude in Bogotá it was expected that $\mathrm{SaO}_{2}$ would be lower than in infants at lower altitudes [21, 22]. Neonates who fell under this definition were considered as cases. Since we used an epidemiological definition for BPD and we did not have the radiographic findings to confirm BPD cases, to avoid misclassification bias "controls" were all infants who were not receiving supplemental oxygen at $36 \mathrm{wPMA}$ and had required $<20$ days of oxygen supplementation or had not required oxygen supplementation at all. Cases and controls were compared to identify risk factors associated with BPD. Relevant exposure data included maternal and infant perinatal characteristics, infant postnatal diagnosis, and respiratory management practices. Preterm premature rupture of membranes (PPROM) was defined as $>12$ hours, use of antenatal steroids as the administration of a complete course (two $12 \mathrm{mg}$ intramuscular doses of betamethasone within 24 hours) of maternal steroids at least 24 hours before delivery, confirmed chorioamnionitis as a positive amniotic fluid culture, and suspected chorioamnionitis as maternal fever during labor and fetal tachycardia. Gestational age (GA) at birth was estimated using the last menstrual period date; when GA was inconsistent with the physical examination, the Ballard score was used [23]. Small for gestational age (SGA) was defined as a birth weight $<10$ th percentile for age [24]. PDA was confirmed by echocardiography and subsequently treated with indomethacin or surgical ligation. Sepsis was defined as a clinical deterioration with temperature instability, recurrent apnea, and at least one of the following indications of altered organ function: lethargy, hypoxemia, and increased serum lactate level [25]. Grades III and IV IVH were identified by head ultrasound using the Papile et al. criteria [26]. Air leak syndrome included radiological evidence of pneumothorax, pneumomediastinum, or pulmonary interstitial emphysema. The Score for Neonatal Acute Physiology (SNAP II) on the day of admission was calculated from clinical data collected prospectively during the first day of life [27]. Respiratory management variables included type of ventilatory support received: (a) only $n C P A P$ when infants were placed on nCPAP without subsequent mechanical ventilation (MV) during their NICU stay, (b) $n C P A P+M V$ when infants on nCPAP met treatment failure criteria and required MV. Length of $M V$ was categorized in $\geq 7$ days or $<7$ days. Exposure to surfactant was divided into three categories: (a) no surfactant exposure included all infants who did not receive surfactant during the trial, (b) very early surfactant included infants administered surfactant within the first hour of life, and (c) rescue surfactant included infants administered surfactant after the first hour of life. Exposure to oxygen supplementation was measured by fraction of inspired oxygen $\left(\mathrm{FIO}_{2}\right)$ registered daily.

Demographic and clinical characteristics of the study population were summarized. To identify differences in the distribution of studied variables between cases and controls, the statistical analysis included hypothesis testing using the Pearson Chi-square test for categorical variables and the Students $t$-test for continuous variables. To explore associations between studied variables and BPD, crude and adjusted odds ratios (ORs) with 95\% confidence intervals (95\% CIs) were estimated in a bivariate analysis, followed by a multivariate regression analysis using a log-binomial generalized linear model that included all significant variables in a manual 
TABLE 1: Demographic characteristics of population studied by cases and controls.

\begin{tabular}{|c|c|c|c|c|}
\hline & \multirow{3}{*}{$\begin{array}{l}\text { All infants } \\
n=212\end{array}$} & \multicolumn{3}{|c|}{ BPD } \\
\hline & & Cases & Controls & $P$ \\
\hline & & $n=64$ & $n=148$ & \\
\hline Maternal age, $y$, mean $\pm(S D)$ & $27.2(6.5)$ & $26.1(6.0)$ & $27.3(6.6)$ & 0.205 \\
\hline Male $(\%)$ & $108(50.9)$ & $34(53.1)$ & $74(50.0)$ & 0.676 \\
\hline Gestational age at birth, wPMA, mean $\pm(\mathrm{SD})$ & $29.2(1.3)$ & $28.6(1.2)$ & $29.3(1.3)$ & 0.001 \\
\hline Birth weight, grams, mean \pm (SD) & $1271.2(283.3)$ & $1155.3(263.0)$ & $1306.5(284.2)$ & 0.000 \\
\hline SGA, $n(\%)$ & $12(5.7)$ & $3(4.7)$ & $9(6.1)$ & 0.688 \\
\hline APGAR score 5 min, median (IR) & $9(8-9)$ & $9(8-9)$ & $9(8-9)$ & 0.228 \\
\hline SNAP-II, score, mean $\pm(\mathrm{SD})$ & $5.7(9)$ & $7.6(10.5)$ & $5.4(8.6)$ & 0.102 \\
\hline
\end{tabular}

BPD: bronchopulmonary dysplasia, wPMA: weeks postmenstrual age, SGA: small for gestational age, SNAP-II: score for neonatal acute physiology, SD: standard deviation, IR: Interquartile range (difference between the value at $75 \%$ of cases, and the value at $25 \%$ of cases).

forward stepwise approach. The Wilcoxon rank sum test was used to compare the median values between groups and the Generalized Cochrane Mantel-Haenszel test to assess the statistical significance of associations between categorical variables.

To determine differences in population characteristics or in respiratory management variables during the first week of life that could explain the differences in BPD rates among cities, a descriptive analysis of selected variables was performed by city of birth, followed by a bivariate analysis to identify variables mainly associated with BPD in each city. To identify possible differences in the distribution of studied variables between three cities among cases of BDP, the statistical analysis included hypothesis testing using the Pearson Chi-square test for categorical variables and the Analysis of Variance or Kruskall Wallis test for continuous variables. Multivariate analyses were then conducted to explore associations between city of birth and BPD while controlling for differences in population demographic characteristics, postnatal diagnosis, and respiratory management variables during the first seven days of life. With the final sample of cases and controls, the power calculation for the research hypothesis tested was $82 \%$, according to the following parameters: incidence of BPD 30\%, type I error probability of 0.05 , and expected size effect (odds ratio, OR) of 2.0. Results of all multivariate analyses are expressed as odds ratios (ORs) with their corresponding 95\% CI. All analyses were carried out using the SAS program (SAS Institute, Cary, NC).

\section{Results}

A total of $216(77.42 \%)$ infants survived to $36 \mathrm{wPMA}$, and four were excluded from the analysis due to missing data or because they did not meet the case or control definitions. The final analysis included 212 infants; 64 (30\%) met the definition of BPD.

3.1. Identification of Risk Factors for BPD in the Whole Population. Mean GA and birth weight were lower in the BPD group (Table 1); no differences were observed in other perinatal characteristics between cases and controls. Tables 2 and 3 present the results of bivariate analysis controlling for GA at birth. Use of rescue surfactant, no surfactant exposure, diagnosis of PDA, chorioamnionitis, and confirmed or suspected PPROM were variables independently associated to BPD.

Following a stepwise forward approach, the initial logistics model showed that PPROM, nCPAP $+M V$, no surfactant exposure, rescue surfactant, PDA, median daily $\mathrm{FIO}_{2}$ (sub index is not allowed in this website), and sepsis were associated with a higher risk of BPD. When city of birth was introduced into the model as a control variable, we observed that median daily $\mathrm{FIO}_{2}$ and PDA lost statistical significance, suggesting the presence of an interaction. We conducted a series of multivariate models testing for interactions between the variables: city of birth, median daily $\mathrm{FIO}_{2}$; sepsis and PDA. The only significant interaction observed was between the variables "Bogotá as city of birth" and "median daily $\mathrm{FIO}_{2}$ " The results of the final logistic regression model controlling this interaction are showed in Table 4. "Bogotá as city of birth" was the most significant independent variable associated with BPD; other variables associated with BPD in the initial model remained significantly associated (Table 4).

3.2. BPD Rates and City-Specific Differences. The distribution of maternal and infant perinatal characteristics, infant postnatal diagnosis, and respiratory management practices in the first seven days of the infants' life was similar in all cities (Table 5); however, there are statistically significant differences between cases and controls among cities.

The proportion of BPD cases in infants whose mothers received antenatal steroids was significantly higher in Bogotá than in any other city. In Bucaramanga, infants with BPD had lower birth weights than infants in Cali or Bogotá. Infants born in Bogotá that required MV after nCPAP ( $n C P A P+$ $M V$ ) and those treated with rescue surfactant or no surfactant exposure had a higher incidence of BPD compared to infants of similar characteristics born in Cali or Bucaramanga. Infants born in Bogotá had higher median values of daily $\mathrm{FIO}_{2}$ compared to Bucaramanga and Cali, and infants diagnosed with BPD in Bogotá received higher concentrations of daily supplemental oxygen than controls, while BPD cases in Cali and Bucaramanga did not. 
TABLE 2: Distribution of maternal characteristics by BPD status.

\begin{tabular}{|c|c|c|c|c|c|c|c|}
\hline \multirow[b]{2}{*}{ Characteristics } & \multirow{2}{*}{$\begin{array}{l}\text { All infants } \\
n=212\end{array}$} & \multicolumn{6}{|c|}{$\mathrm{BPD}$} \\
\hline & & Cases & Controls & $P$ & $\mathrm{OR}^{*}$ & \multicolumn{2}{|c|}{$95 \% \mathrm{CI}$} \\
\hline \multicolumn{8}{|l|}{ Maternal characteristics } \\
\hline \multicolumn{8}{|l|}{ Mean age, $n(\%)$} \\
\hline$<18$ years & $15(7.1)$ & $5(7.8)$ & $10(6.8)$ & \multirow{3}{*}{0.959} & 1.08 & 0.38 & 3.08 \\
\hline $18-35$ years & $173(81.6)$ & $52(81.3)$ & $121(81.8)$ & & 1.00 & & \\
\hline$>35$ years & $24(11.3)$ & $7(10.9)$ & $17(11.5)$ & & 0.97 & 0.50 & 1.88 \\
\hline \multicolumn{8}{|l|}{ Type of delivery, $n(\%)$} \\
\hline Vaginal & $35(16.5)$ & $12(18.8)$ & $23(15.5)$ & \multirow{2}{*}{0.563} & 1.00 & & \\
\hline Cesarean & $177(83.5)$ & $52(81.3)$ & $125(84.5)$ & & 0.86 & 0.51 & 1.43 \\
\hline Multiple gestation $n(\%)$ & $22(10.4)$ & $6(9.4)$ & $16(10.8)$ & 0.753 & 0.89 & 0.44 & 1.83 \\
\hline Antenatal steroids, $n(\%)$ & $184(86.8)$ & $56(87.5)$ & $128(86.5)$ & 0.072 & 1.32 & 0.63 & 2.75 \\
\hline PPROM, $n(\%)$ & $76(35.8)$ & $30(46.9)$ & $46(31.2)$ & 0.028 & 1.58 & 1.06 & 2.36 \\
\hline \multicolumn{8}{|l|}{ Duration of rupture of membranes, $n(\%)$} \\
\hline$<24 \mathrm{~h}$ & $35(46.1)$ & $12(40.0)$ & $23(50.0)$ & \multirow{4}{*}{0.275} & 1.00 & & \\
\hline $24-48 \mathrm{~h}$ & $14(18.4)$ & $5(16.7)$ & $9(19.6)$ & & 0.76 & 0.14 & 4.02 \\
\hline $48-72 \mathrm{~h}$ & $8(10.5)$ & $2(6.7)$ & $6(13.0)$ & & 1.76 & 0.52 & 5.94 \\
\hline$>72 \mathrm{~h}$ & $19(25.0)$ & $11(36.7)$ & $8(17.4)$ & & 1.04 & 0.45 & 2.41 \\
\hline Chorio confirmed, $n(\%)$ & $11(5.2)$ & $4(6.5)$ & $7(4.7)$ & 0.647 & 1.22 & 0.54 & 2.74 \\
\hline Chorio confirmed or suspected, $n(\%)$ & $39(18.4)$ & $17(26.6)$ & $22(14.9)$ & 0.044 & 1.60 & 1.04 & 2.47 \\
\hline
\end{tabular}

Chorio: chorioamnionitis, PPROM: preterm premature rupture of membranes.

In relation to postnatal diagnosis during the first 7 days of life, the diagnosis of PDA was higher in Bucaramanga, but the largest proportion of BPD cases in infants with PDA was observed in Bogotá. The diagnosis of sepsis was also more frequent in Bucaramanga and Cali, but the largest proportion of BPD cases among infected infants was seen in Bogotá, followed by Cali and Bucaramanga $(P<0.0001)$.

Bivariate analysis stratified by city of birth (Table 5) showed the following: for infants born in Bogotá, the diagnosis of sepsis in the first seven days of life and the incremental levels of daily median $\mathrm{FIO}_{2}$ as variables mainly associated with a higher risk of BPD; for infants born in Cali, air leak syndrome, $P P R O M$, and $P D A$ in the first seven days of life were the variables associated with $\mathrm{BPD}$, while in Bucaramanga $P P R O M$ was the only significant risk factor associated with BPD.

To explore for associations between city of birth and BPD, we generated a logistics model controlling potential confounders. Multivariate results are shown in Table 6; Bogotá as city of birth was strongly associated with an increased risk of developing BPD (OR 1.82 95\%CI 1.31-2.53). Other variables in the model associated with a higher risk of BPD were rescue surfactant, no surfactant exposure, $n C P A P+M V$, median daily $\mathrm{FIO}_{2}, P D A$, and sepsis.

\section{Discussion}

In this study population, the incidence of BPD was $30 \%$. This rate is higher than the average rates reported in populations with similar gestational ages in developed countries $[1,2,5-$ 7]. Our results showed that infants born in Bogotá had nearly twice the risk of developing BPD than infants born in Bucaramanga or Cali, independently of differences in maternal, infant, and therapeutic risk factors. Additionally, Bogotá showed the highest rates of BPD cases associated with the presence of air leak syndrome, exposure to MV, rescue surfactant or no surfactant exposure, PDA, and sepsis, when compared to other cities. It also had the highest values of daily supplemental oxygen to reach the target $\mathrm{SaO}_{2}$ among all the cities. Exposure to antenatal steroids did not appear to protect infants born in Bogotá from developing BPD.

Because Bogotá is located a higher altitude (more than 2600 masl) than Bucaramanga and Cali, these results could suggest that altitude may play an important role in the pathogenesis of BPD in Colombia. There are few publications on altitude-related disease and pulmonary hemodynamics in pediatric populations, and the altitude where an infant is born has not clearly been proven to be associated with the development of BPD. The effect of living at high altitudes (>2500 masl) on lung diffusion capacity and pulmonary hemodynamics has been described in highland children [28]. As a result of the low partial pressure of oxygen in the environment, oxygen uptake into the lungs is enhanced by increases in minute ventilation, lung compliance, and pulmonary diffusion. The decreased partial pressure of oxygen in the lungs of highland children has also been associated with higher pulmonary artery pressures [28-31]. Several investigators have also found that functional closure of 
TABLE 3: Distribution of infant characteristics by BPD status.

\begin{tabular}{|c|c|c|c|c|c|c|c|}
\hline \multirow{2}{*}{ Characteristics } & \multirow{2}{*}{ All infants } & \multicolumn{6}{|c|}{ BPD } \\
\hline & & $\begin{array}{c}\text { Cases } \\
n=64\end{array}$ & $\begin{array}{c}\text { Controls } \\
n=48\end{array}$ & $P$ & $\mathrm{OR}^{*}$ & \multicolumn{2}{|c|}{$95 \%$ CI } \\
\hline \multicolumn{8}{|l|}{ Sex, $n(\%)$} \\
\hline Male & $108(50.9)$ & $34(53.1)$ & $74(50.0)$ & \multirow{2}{*}{0.676} & 1.10 & \multirow[t]{2}{*}{0.73} & \multirow[t]{2}{*}{1.66} \\
\hline Female & $104(49.1)$ & $30(46.9)$ & $74(50.0)$ & & 1.00 & & \\
\hline \multicolumn{8}{|l|}{ Gestational age, $n(\%)$} \\
\hline 27-29 weeks & $114(53.8)$ & $40(62.5)$ & $74(50.0)$ & \multirow{2}{*}{0.094} & 1.43 & \multirow[t]{2}{*}{0.93} & \multirow[t]{2}{*}{2.20} \\
\hline 30-31 weeks & $98(46.2)$ & $24(37.5)$ & $74(50.0)$ & & 1.00 & & \\
\hline \multicolumn{8}{|l|}{ Birth weight, $n(\%)$} \\
\hline$<1000 \mathrm{~g}$ & $37(17.4)$ & $14(21.9)$ & $23(15.5)$ & \multirow{3}{*}{0.301} & 1.70 & 0.86 & 3.38 \\
\hline $1000-1500 \mathrm{~g}$ & $130(61.3)$ & $40(62.5)$ & $90(60.8)$ & & 1.30 & \multirow[t]{2}{*}{0.76} & \multirow[t]{2}{*}{2.53} \\
\hline$>1500 \mathrm{~g}$ & $45(21.2)$ & $10(15.6)$ & $35(23.6)$ & & 1.00 & & \\
\hline SGA $n(\%)$ & $12(5.7)$ & $3(4.7)$ & $9(6.1)$ & 0.687 & 0.82 & 0.30 & 2.23 \\
\hline Apgar score $<5$ at $5 \min , n(\%)$ & $2(0.94)$ & $0(0.0)$ & $2(1.35)$ & 0.350 & 1.00 & 0.82 & 1.21 \\
\hline \multicolumn{8}{|l|}{ Mean SNAP II, $n(\%)$} \\
\hline 0 to 10 & $143(67.4)$ & $102(68.9)$ & $41(64.0)$ & \multirow{3}{*}{0.381} & & & \\
\hline 11 to 19 & $54(5.5)$ & $36(24.3)$ & $18(28.1)$ & & & & \\
\hline$>20$ & $15(7.1)$ & $10(6.8)$ & $5(7.2)$ & & & & \\
\hline $\mathrm{PDA}(\%)$ & $64(30.2)$ & $30(46.9)$ & $34(23.0)$ & 0.001 & 2.04 & 1.38 & 3.03 \\
\hline Air Leak Syndrome (\%) & $12(5.7)$ & $6(9.4)$ & $6(4.0)$ & 0.124 & 1.72 & 0.94 & 3.16 \\
\hline Sepsis $(\%)$ & $109(51.4)$ & $39(60.9)$ & $70(47.3)$ & 0.068 & 1.47 & 0.98 & 2.38 \\
\hline IVH grade III or IV (\%) & $4(1.90)$ & $1(1.6)$ & $3(2.0)$ & 0.820 & 0.83 & 0.15 & 4.56 \\
\hline \multicolumn{8}{|l|}{ Ventilatory Support (\%) } \\
\hline nCPAP only & $162(76.4)$ & $37(57.8)$ & $125(84.5)$ & \multirow[t]{3}{*}{0.000} & 1 & & \\
\hline nCPAP + MV & $50(23.6)$ & $27(42.2)$ & $23(15.5)$ & & 2.36 & 1.61 & 3.46 \\
\hline $\mathrm{LOMV} \geq 7$ days $(\%)^{*}$ & $15(30.0)^{*}$ & $8(29.6)$ & $7(30.4)$ & & 1.12 & 0.68 & 1.84 \\
\hline \multicolumn{8}{|l|}{ Use of Surfactant (\%) } \\
\hline Very early & $106(50.0)$ & $20(31.2)$ & $86(58.1)$ & \multirow[t]{3}{*}{0.000} & 1 & & \\
\hline Rescue & $27(12.7)$ & $18(28.1)$ & $9(6.1)$ & & 6.16 & 2.53 & 15.02 \\
\hline No surfactant & $79(37.3)$ & $26(40.6)$ & $53(35.8)$ & & 3.53 & 2.19 & 5.69 \\
\hline $\mathrm{FIO}_{2}$ (median values) & 0.53 & 0.52 & 0.52 & 0.102 & & & \\
\hline
\end{tabular}

Deaths before 36 wPMA are excluded. BPD: bronchopulmonary dysplasia, SGA: small for gestational age, SNAP-II: score for neonatal acute physiology, nCPAP: nasal continuous positive airway pressure, MV: mechanical ventilation, LOMV: length of mechanical ventilation, PDA: patent ductus arteriosus.

TABLE 4: Multivariate analysis predicting BPD for the entire cohort controlled by city of birth $N=212$.

\begin{tabular}{|c|c|c|c|}
\hline Variables & OR & \multicolumn{2}{|c|}{$95 \%$ CI } \\
\hline Preterm premature rupture of membranes & 1.17 & 1.05 & 1.31 \\
\hline Ventilatory support (nCPAP + MV) & 1.18 & 1.01 & 1.38 \\
\hline Use of surfactant (rescue) & 1.63 & 1.28 & 2.08 \\
\hline Use of surfactant (no surfactant) & 1.37 & 1.12 & 1.67 \\
\hline City of birth (Bogotá) ${ }^{1}$ & 1.9 & 1.46 & 2.49 \\
\hline $\mathrm{FIO}_{2}^{1}$ (median value) & 1.58 & 1.11 & 2.26 \\
\hline PDA & 1.14 & 1.01 & 1.28 \\
\hline Sepsis & 1.12 & 1.01 & 1.26 \\
\hline
\end{tabular}

the ductus arteriosus in the newborn is delayed at high altitudes as a consequence of increased pulmonary vascular pressures [32-35]. The transition from oxygenation via the placenta to oxygenation across the formerly fluid-filled lungs is especially precarious in a low-oxygen environment [36]; with the onset of ventilation immediately after birth, the oxygen tension in the alveolus and pulmonary capillaries of neonates may not increase as expected, resulting in postnatal persistence of fetal circulation [36]. In the absence of pulmonary disorders, normal neonates may experience frequent episodes of arterial oxygen desaturations and hypoxia during the first week of life. Studies comparing healthy infants born at high altitudes ( $>3100$ masl) with infants born at sea level have shown that a week after birth the $\mathrm{SaO}_{2}$ of high-altitude infants declines, whereas $\mathrm{SaO}_{2}$ gradually rises after birth or remains constant over time in infants born at sea level [32]. As a result of these events, it is possible that premature infants born at high altitudes have a prolonged transition period and early dependency on high concentrations of oxygen and ventilatory support compared to their counterparts born at lower altitudes. The presence of RDS would enhance ventilation perfusion mismatch leading to more hypoxia, oxygen dependency, oxidative stress, and higher levels of ventilatory support, increasing the risk and severity of BPD. It is also possible that the observed dependency on supplemental oxygen in our population of premature infants is the result of physiological oxygen dependency and not BPD. This may 


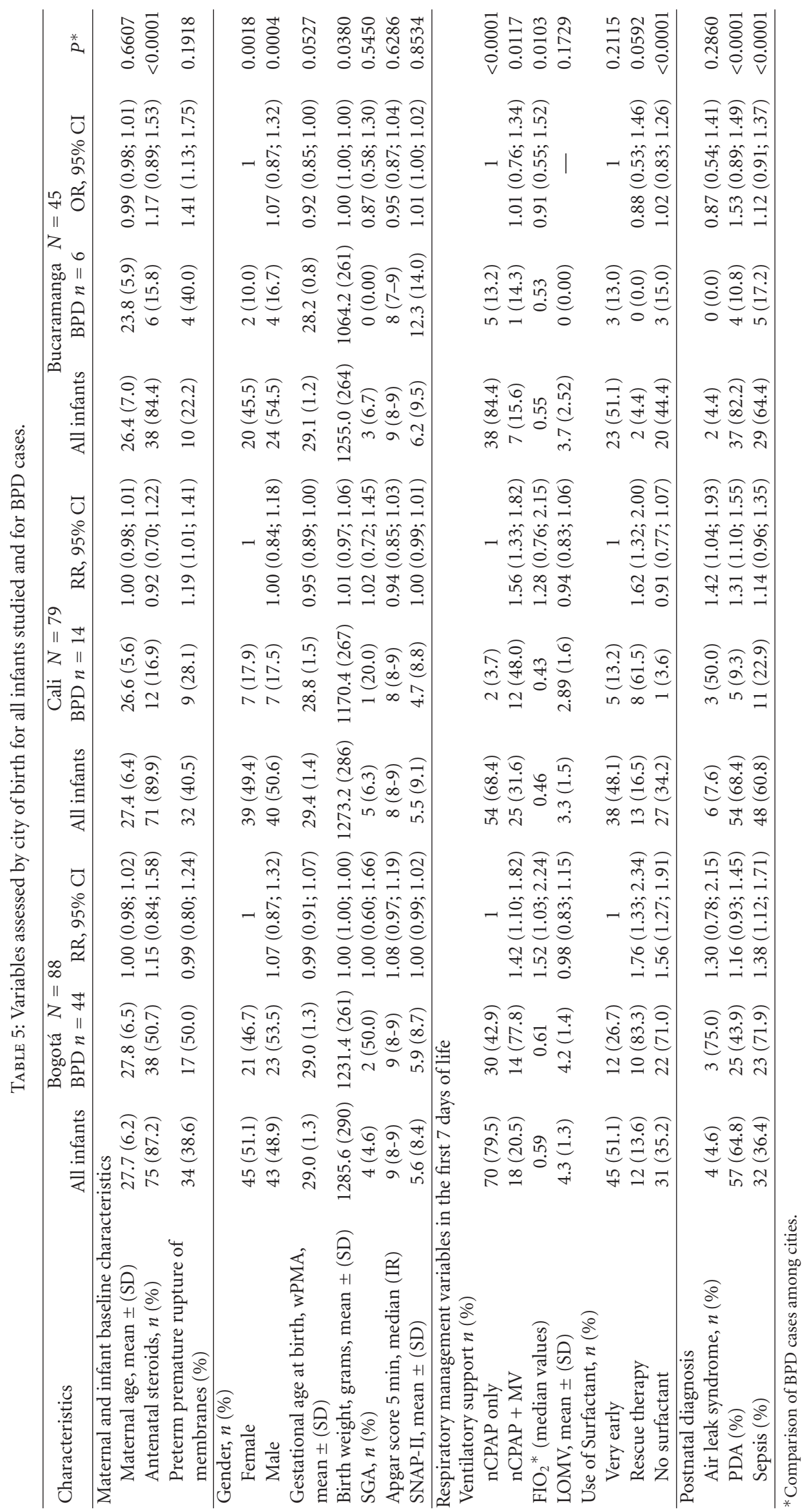


TABLE 6: Multivariate analysis exploring associations between BPD and city of birth $(n=208)$.

\begin{tabular}{lccc}
\hline & OR & \multicolumn{2}{c}{$95 \%$ CI } \\
\hline City of birth (Bogotá) $^{1}$ & 1.82 & 1.31 & 2.53 \\
City of birth (Cali) & 0.80 & 0.46 & 1.39 \\
Gender (male) & 1.04 & 0.93 & 1.15 \\
Gestational age (wPMA) & 1.02 & 0.97 & 1.07 \\
Birth weight & 1.00 & 1.00 & 1.00 \\
Antenatal steroids & 1.00 & 0.84 & 1.18 \\
Preterm premature rupture of membranes & 1.18 & 1.05 & 1.33 \\
Ventilatory support (nCPAP + MV) & 1.15 & 1.02 & 1.38 \\
Use of Surfactant (rescue) & 1.36 & 1.10 & 1.68 \\
Use of Surfactant (no surfactant) & 1.19 & 1.06 & 1.33 \\
FIO ${ }_{2}$ (median value) & 1.54 & 1.10 & 2.16 \\
Air Leak syndrome & 1.54 \\
PDA & 0.96 & 0.75 & 1.24 \\
Sepsis & 1.13 & 1.00 & 1.29 \\
\hline
\end{tabular}

${ }^{1}$ OR adjusted by interaction term $\mathrm{City} \times \mathrm{FIO}_{2}$ (Lincom statistics). nCPAP: nasal continuous airway pressure, MV: mechanical ventilation, PDA: patent ductus arteriousus.

be due to the fact that the definition used for BPD does not take into account clinical or radiographic changes.

The association between PDA and BPD has previously been documented $[4,12,37]$. It is also possible that the prolonged closure of the PDA may play a role in the pathogenesis of BPD, especially as the pulmonary vascular resistance begins to drop, but we cannot answer this question because we did not assess the duration of the PDA, the presence of pulmonary hypertension, or the presence of pulmonary edema or hemorrhage in this population of infants. Likewise, we did not assess the effect of fluid intake on the development of PDA and BPD because this information was not collected in the initial trial $[7,14,37,38]$. Previous studies have demonstrated the relationship between the presence of lateonset sepsis, PDA, and the development of BPD [12, 37]. Our study suggests that altitude enhances their negative effects and other traditional risk factors associated with BPD [38]. In our population, $P P R O M$ was found to be a significant risk factor for BPD while chorioamnionitis was not. This finding could be explained in part because amniocentesis and amniotic culture fluids were not taken routinely in all participating centers as part of the workup for suspected chorioamnionitis in mothers with preterm labor or premature rupture of membranes. The association of BPD with preterm labor and PPROM has been well documented in the medical literature [39].

Finally, our study emphasizes the need to minimize MV exposure and offer surfactant replacement therapy within the first hour of life to infants with RDS on NCPAP in order to decrease the incidence of BPD in this population $[20,40,41]$.

To summarize, this study suggests that altitude may be an important risk factor associated with increased supplemental oxygen dependency and the development of BPD. Future studies need to determine whether altitude plays a role in the pathophysiology of BPD or if it is just a marker for physiologic oxygen demands. They also need to use a more accurate definition for BPD, measuring not only the need of supplemental oxygen but also the radiographic findings and clinical signs.

\section{Disclosure}

Authors declare that material of this paper is original research, it has not been previously published, and it has not been submitted for publication elsewhere while under consideration.

\section{Conflict of Interests}

Authors declare that they do not have any competing financial interests in relation to the work described in this paper. M. A. Rojas, J. M. Lozano, M. A. Rondón, and M. X. Rojas have participated as authors of independent trials supported in part by Abbott Laboratories, SensorMedics Corporation, and Fisher \& Paykel Healthcare. Mario Augusto Rojas has acted as a consultant for Fisher \& Paykel Healthcare.

\section{Authors' Contribution}

M. X. Rojas and M. A. Rojas set together the objectives. M. X. Rojas designed the methodological approach of the study, was responsible of execution of the study and made the data interpretation and writing the paper, also the final review and edition of version to be published. M. A. Rojas reviewed and edited the manuscript, made substantive intellectual contributions to the paper, and gave the final approval of the version to be published. Juan Manual Lozano was responsible for revision of the manuscript and made substantive intellectual contributions to this process and gave the approval of the version to be published. M. A. Rondón conducted the data analysis and made substantive intellectual contributions to the manuscript. L. P. Charry contributed to data collection and revised the final manuscript to be published.

\section{Acknowledgments}

The authors thank Sergio Mario Castro, Research Assistant at the Clinical Epidemiology Department of the PontificiaUniversidad Javeriana for his contribution during the study process. They also thank all the principal investigators from the Colombian Neonatal Research Network (CNRN) for allowing them to use their data base for this study. The study protocol was approved by the Institutional Review Board of the Pontificia Universidad Javeriana. This study was conducted as part of the Doctoral Program in "Salud Pública y Metodología de la Investigación Biomédica/Public Health and Biomedical Investigation Methodology" of the Department of Pediatrics and Preventive Medicine, School of Medicine, Universitat Autònoma de Barcelona, Spain. Professor M. X. Rojas is a Ph.D. candidate in this program. This research was funded by Pontificia Universidad Javeriana and Vanderbilt University. The Wake Forest University and the Florida International University supported the time dedicated by two authors. 


\section{References}

[1] A. H. Jobe and E. Bancalari, "Bronchopulmonary dysplasia," American Journal of Respiratory and Critical Care Medicine, vol. 163, no. 7, pp. 1723-1729, 2001.

[2] R. A. Ehrenkranz, M. C. Walsh, B. R. Vohr et al., "Validation of the National Institutes of health consensus definition of bronchopulmonary dysplasia," Pediatrics, vol. 116, no. 6, pp. 13531360, 2005.

[3] A. A. Hislop, J. S. Wigglesworth, R. Desai, and V. Aber, "The effects of preterm delivery and mechanical ventilation on human lung growth," Early Human Development, vol. 15, no. 3, pp. 147-164, 1987.

[4] M. Palta, D. Gabbert, M. R. Weinstein et al., "Multivariate assessment of traditional risk factors for chronic lung disease in very low birth weight neonates," Journal of Pediatrics, vol. 119, no. 2, pp. 285-292, 1991.

[5] L. J. Van Marter, M. Pagano, E. N. Allred, A. Leviton, and K. C. K. Kuban, "Rate of bronchopulmonary dysplasia as a function of neonatal intensive care practices," Journal of Pediatrics, vol. 120, no. 6, pp. 938-946, 1992.

[6] T. Farstad and D. Bratlid, "Incidence and prediction of bronchopulmonary dysplasia in a cohort of premature infants," Acta Paediatrica, International Journal of Paediatrics, vol. 83, no. 1, pp. 19-24, 1994.

[7] D. D. Marshall, M. Kotelchuck, T. E. Young, C. L. Bose, P. A. C. Lauree Kruyer, and T. M. O'Shea, "Risk factors for chronic lung disease in the surfactant era: a North Carolina population-based study of very low birth weight infants," Pediatrics, vol. 104, no. 6, pp. 1345-1350, 1999.

[8] P. J. Sanchez and J. A. Regan, "Ureaplasma urealyticum colonization and chronic lung disease in low birth weight infants," Pediatric Infectious Disease Journal, vol. 7, no. 8, pp. 542-546, 1988.

[9] E. E. L. Wang, H. Frayha, J. Watts et al., "Role of Ureaplasma urealyticum and other pathogens in the development of chronic lung disease of prematurity," Pediatric Infectious Disease Journal, vol. 7, no. 8, pp. 547-551, 1988.

[10] A. H. Jobe and M. Ikegami, "Antenatal infection/inflammation and postnatal lung maturation and injury," Respiratory Research, vol. 2, no. 1, pp. 27-32, 2001.

[11] L. J. Van Marter, O. Dammann, E. N. Allred et al., "Chorioamnionitis, mechanical ventilation, and postnatal sepsis as modulators of chronic lung disease in preterm infants," Journal of Pediatrics, vol. 140, no. 2, pp. 171-176, 2002.

[12] M. A. Rojas, A. Gonzalez, E. Bancalari, N. Claure, C. Poole, and G. Silva-Neto, "Changing trends in the epidemiology and pathogenesis of neonatal chronic lung disease," Journal of Pediatrics, vol. 126, no. 4, pp. 605-610, 1995.

[13] T. Farstad and D. Bratlid, "Pulmonary effects of closure of patent ductus arteriosus in premature infants with severe respiratory distress syndrome," European Journal of Pediatrics, vol. 153, no. 12, pp. 903-905, 1994.

[14] L. J. Van Marter, A. Leviton, E. N. Allred, M. Pagano, and K. C. K. Kuban, "Hydration during the first days of life and the risk of bronchopulmonary dysplasia in low birth weight infants," Journal of Pediatrics, vol. 116, no. 6, pp. 942-949, 1990.

[15] A. R. Spitzer, W. W. Fox, and M. Delivoria-Papadopoulos, "Maximum diuresis - a factor in predicting recovery from respiratory distress syndrome and the development of bronchopulmonary dysplasia," Journal of Pediatrics, vol. 98, no. 3, pp. 476-479, 1981.

[16] B. A. Darlow and P. J. Graham, "Vitamin A supplementation to prevent mortality and short and long-term morbidity in very low birthweight infants," Cochrane Database of Systematic Reviews, no. 4, Article ID CD000501, 2007.

[17] K. L. Watterberg, J. S. Gerdes, C. H. Cole et al., "Prophylaxis of early adrenal insufficiency to prevent bronchopulmonary dysplasia: a multicenter trial," Pediatrics, vol. 114, no. 6, pp. 1649-1657, 2004.

[18] P. Kwinta, M. Bik-Multanowski, Z. Mitkowska, T. Tomasik, M. Legutko, and J. J. Pietrzyk, "Genetic risk factors of bronchopulmonary dysplasia," Pediatric Research, vol. 64, no. 6, pp. 682-688, 2008.

[19] J. L. Tapia, D. Agost, A. Alegria et al., "Bronchopulmonary dysplasia: incidence, risk factors and resource utilization in a population of South American very low birth weight infants," Jornal de Pediatria, vol. 82, no. 1, pp. 15-20, 2006.

[20] M. A. Rojas, J. M. Lozano, M. X. Rojas et al., "Very early surfactant without mandatory ventilation in premature infants treated with early continuous positive airway pressure: a randomized, controlled trial," Pediatrics, vol. 123, no. 1, pp. 137-142, 2009.

[21] J. M. Lozano, O. R. Duque, T. Buitrago, and S. Behaine, "Pulse oximetry reference values at high altitude," Archives of Disease in Childhood, vol. 67, no. 3, pp. 299-301, 1992.

[22] S. Balasubramanian, N. Suresh, R. Raeshmi, and K. Kaarthigeyan, "Comparison of oxygen saturation levels by pulse oximetry in healthy children aged 1 month to 5 years residing at an altitude of 1500 metres and at sea level," Annals of Tropical Paediatrics, vol. 28, no. 4, pp. 267-273, 2008.

[23] J. L. Ballard, J. C. Khoury, K. Wedig, L. Wang, B. L. EilersWalsman, and R. Lipp, "New Ballard Score, expanded to include extremely premature infants," Journal of Pediatrics, vol. 119, no. 3, pp. 417-423, 1991.

[24] G. F. Gonzales and V. Tapia, "Birth weight charts for gestational age in 63620 healthy infants born in Peruvian public hospitals at low and at high altitude," Acta Paediatrica, International Journal of Paediatrics, vol. 98, no. 3, pp. 454-458, 2009.

[25] M. M. Levy, M. P. Fink, J. C. Marshall et al., "2001 SCCM/ ESICM/ACCP/ATS/SIS international sepsis definitions conference," Critical Care Medicine, vol. 31, no. 4, pp. 1250-1256, 2003.

[26] L. A. Papile, J. Burstein, R. Burstein, and H. Koffler, "Incidence and evolution of subependymal and intraventricular hemorrhage: a study of infants with birth weights less than 1,500 gm," Journal of Pediatrics, vol. 92, no. 4, pp. 529-534, 1978.

[27] D. K. Richardson, J. D. Corcoran, G. J. Escobar, and S. K. Lee, "SNAP-II and SNAPPE-II: simplified newborn illness severity and mortality risk scores," Journal of Pediatrics, vol. 138, no. 1, pp. 92-100, 2001.

[28] K. De Meer, H. S. A. Heymans, and W. G. Zijlstra, "Physical adaptation of children to life at high altitude," European Journal of Pediatrics, vol. 154, no. 4, pp. 263-272, 1995.

[29] K. De Meer, R. Bergman, J. S. Kusner, and H. W. A. Voorhoeve, "Differences in physical growth of Aymara and Quechua children living at high altitude in Peru," American Journal of Physical Anthropology, vol. 90, no. 1, pp. 59-75, 1993.

[30] R. Gamboa and E. Marticorena, "Pulmonary arterial pressure in newborn infants in high altitude," Archivos del Instituto de Biologia Andina, vol. 4, no. 2, pp. 55-66, 1971.

[31] F. Sime, N. Banchero, D. Peñaloza, R. Gamboa, J. Cruz, and E. Marticorena, "Pulmonary hypertension in children born and living at high altitudes," American Journal of Cardiology, vol. 11, no. 2, pp. 143-149, 1963.

[32] S. Niermeyer, E. M. Shaffer, E. Thilo, C. Corbin, and L. G. Moore, "Arterial oxygenation and pulmonary arterial pressure 
in healthy neonates and infants at high altitude," Journal of Pediatrics, vol. 123, no. 5, pp. 767-772, 1993.

[33] R. Lavadenz, E. Palmero, F. Loma, and R. Carreon, "Patent ductus arteriosus with pulmonary hypertension," Arquivos Brasileiros de Cardiologia, vol. 47, no. 5, pp. 323-327, 1986.

[34] C. Y. Miao, J. S. Zuberbuhler, and J. R. Zuberbuhler, "Prevalence of congenital cardiac anomalies at high altitude," Journal of the American College of Cardiology, vol. 12, no. 1, pp. 224228, 1988.

[35] S. Niermeyer, "Cardiopulmonary transition in the high altitude infant," High Altitude Medicine and Biology, vol. 4, no. 2, pp. 225-239, 2003.

[36] S. Niermeyer, P. A. Mollinedo, and L. Huicho, "Child health and living at high altitude," Archives of Disease in Childhood, vol. 94, no. 10, pp. 806-811, 2009.

[37] A. Gonzalez, I. R. S. Sosenko, J. Chandar, H. Hummler, N. Claure, and E. Bancalari, "Influence of infection on patent ductus arteriosus and chronic lung disease in premature infants weighing 1000 grams or less," Journal of Pediatrics, vol. 128, no. 4, pp. 470-478, 1996.

[38] G. Rocha, O. Ribeiro, and H. Guimarães, "Fluid and electrolyte balance during the first week of life and risk of bronchopulmonary dysplasia in the preterm neonate," Clinics, vol. 65, no. 7, pp. 663-674, 2010.

[39] B. H. Yoon, R. Romero, K. S. Kim et al., "A systemic fetal inflammatory response and the development of bronchopulmonary dysplasia," American Journal of Obstetrics and Gynecology, vol. 181, no. 4, pp. 773-779, 1999.

[40] R. F. Soll and C. J. Morley, "Prophylactic versus selective use of surfactant in preventing morbidity and mortality in preterm infants," Cochrane Database of Systematic Reviews, no. 2, Article ID CD000510, 2001.

[41] T. P. Stevens, E. W. Harrington, M. Blennow, and R. F. Soll, "Early surfactant administration with brief ventilation vs. selective surfactant and continued mechanical ventilation for preterm infants with or at risk for respiratory distress syndrome," Cochrane Database of Systematic Reviews, no. 4, Article ID CD003063, 2007. 


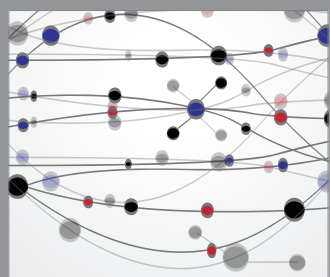

The Scientific World Journal
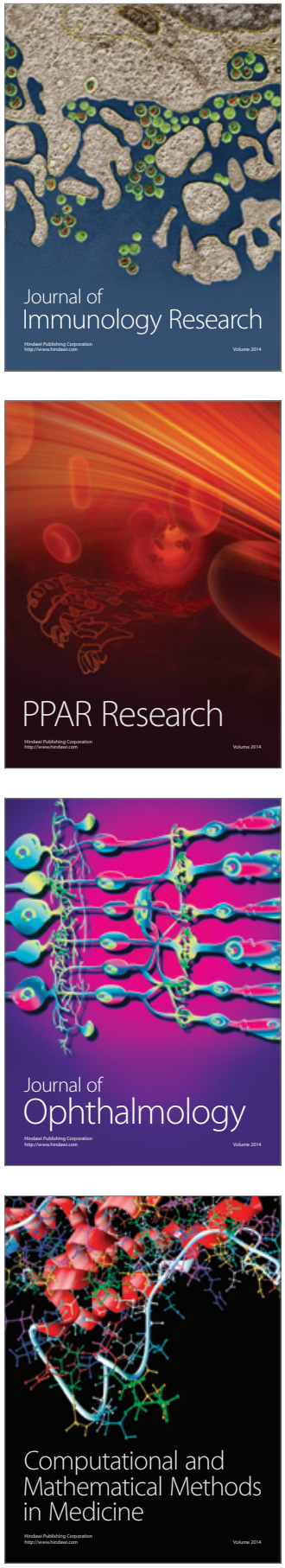

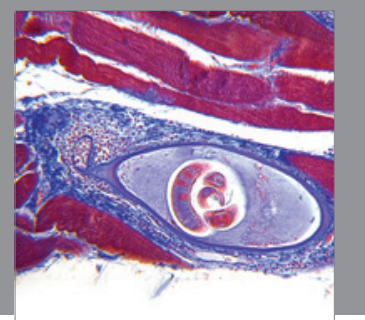

Gastroenterology

Research and Practice
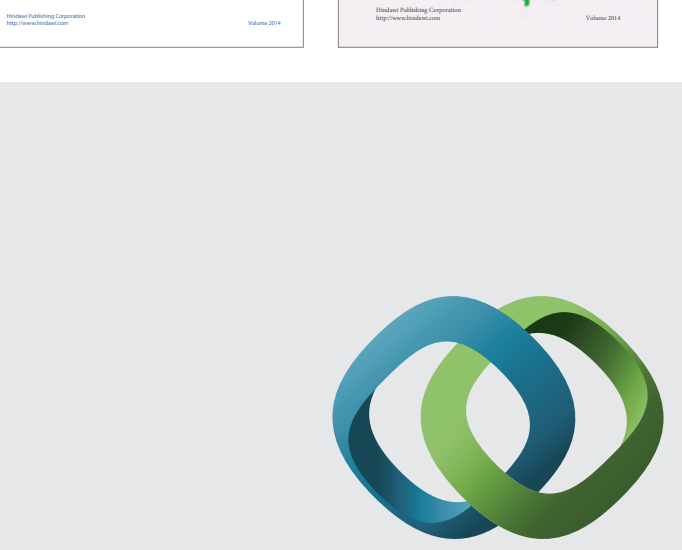

\section{Hindawi}

Submit your manuscripts at

http://www.hindawi.com
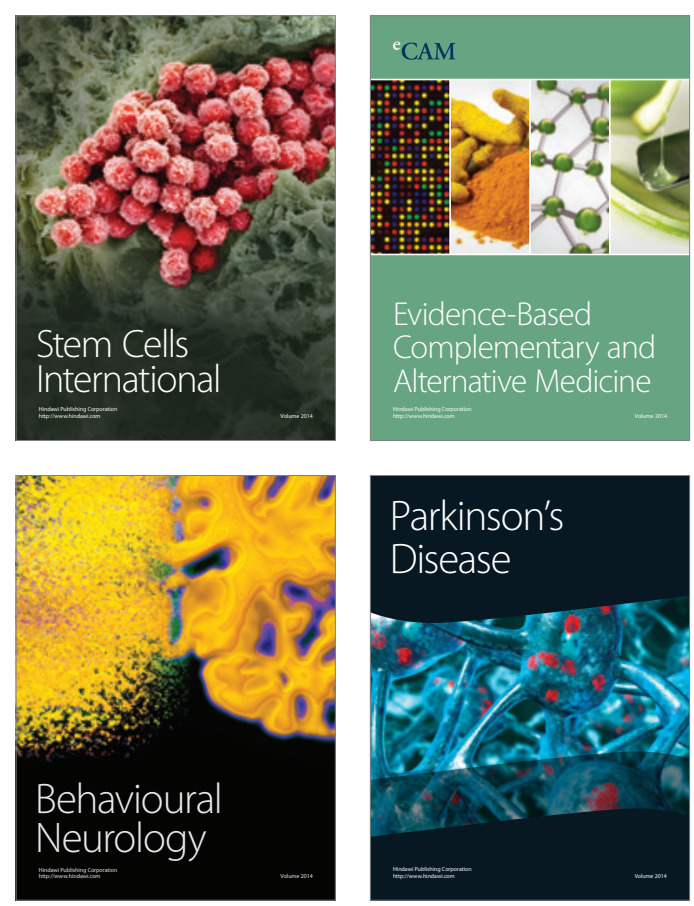

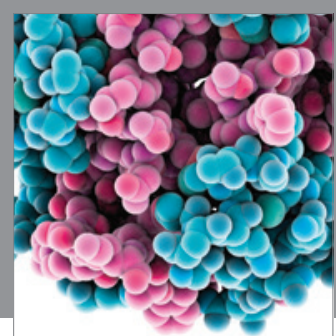

Journal of
Diabetes Research

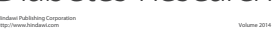

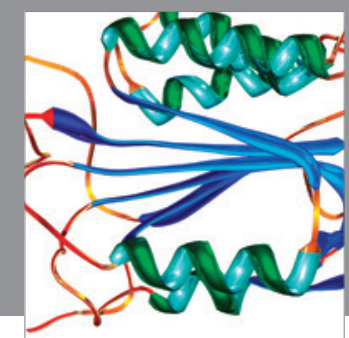

Disease Markers
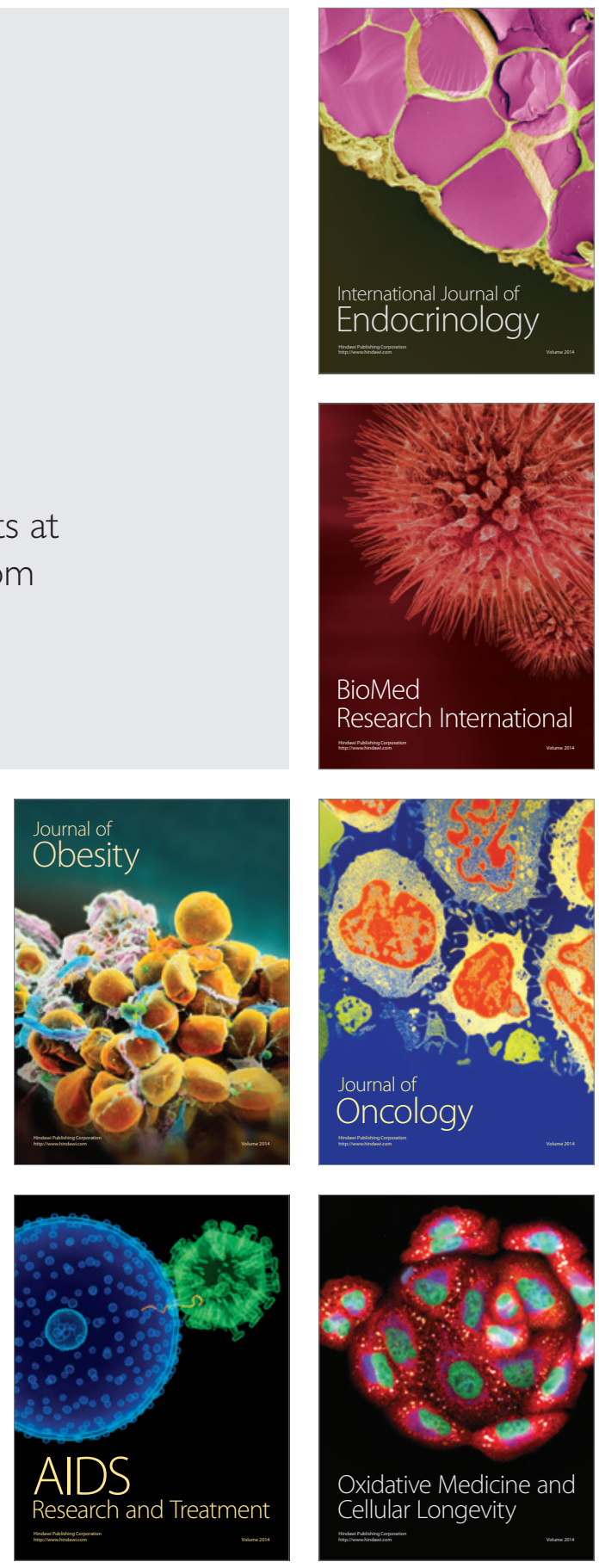